\section{Design of CMOS MEMS Based on Mechanical Resonators Using a RF Simulation Approach}

\author{
Laurent Latorre, Vincent Beroulle, and Pascal Nouet
}

\begin{abstract}
This paper, which is mostly tutorial in nature, deals with the design of CMOS microelectromechanical systems MEMS using standard microelectronic computer-aided design tools. The proposed case study is an on-chip spectrum analyzer with an electronic mixer and a mechanical filter. Based on both analytical modeling and characterization, the filter is described using an analog hardware description language. System level simulations are then performed using a recently released simulation tool that offer new possibilities regarding the analysis of multidomain, multifrequency designs. Presented results include periodic steady state determination, small-signal analysis and noise investigation. The simulations demonstrate the ability of the proposed system to identify the harmonics of a $50-\mathrm{Hz}$ square-wave signal, owing to the selectivity of the mechanical filter.
\end{abstract}

Index Terms-CMOS, microelectromechanical system (MEMS), resonator, simulation.

\section{INTRODUCTION}

Microelectromechanical system (MEMS) fabrication approaches differ depending on the technologies used for transducer manufacturing. When specific technologies incompatible with microelectronic standards (i.e., CMOS) are required for mechanical part fabrication, electronic functions are provided on a separate die using various assembly techniques (hybrid approach). An alternative to hybrid manufacturing is the monolithic integration of MEMS, where both mechanical and electronic functions are located on the same die. This leads to a significant reduction of fabrication costs, although requirements for process compatibility usually limit performance level and application choice. Increasing interest is given to such devices which are intended to address large volume markets, where batch fabrication is the only solution. However, the obtained integrated circuits are so complex that some system-level design automation tools are required. The latter include schematic capture, both systemand circuit-level simulations and layout edition.

The most popular modeling approach for MEMS is based on the use of finite elements models (FEM). These are used whenever analytical solutions are difficult to achieve, as for MEMS with complex geometry or multiple actuation modes. Such models, coupled with an FEM simulator, provide accurate simulation results for various physical domains, such as mechanical, thermal, or magnetic. The price for this accuracy is a long simulation time that is particularly inopportune during the optimization process. Moreover, FEM simulators are not yet easily coupled with mixed-signal electrical simulators. Like the technology computer-aided design (CAD) for transistor-based designs, FEM simulators are efficient and accurate at the device level, whereas other solutions are necessary at the circuit or system level. It then becomes necessary to deal with analytical models that may be integrated in a microelectronic design flow in order to simulate electromechanical behaviors concurrently with MOS transistors described by their electrical models. A recent feature of standard microelectronic CAD tools is the analog HDL language. Any physical-component simulation, together with a mixed-signal processing circuit, is thereby supported [1], [2]. In

Manuscript received May 31, 2002; revised November 13, 2002 and May 20, 2003. This paper was recommended by Associate Editor H. A. Mantooth.

The authors are with the Laboratoire d'Informatique, de Robotique et de Microelectronique de Montpellier, 34392 Montpellier, France (e-mail latorre@lirmm.fr).

Digital Object Identifier 10.1109/TCAD.2004.828113

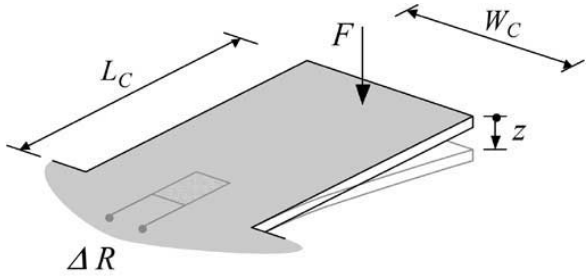

(a)

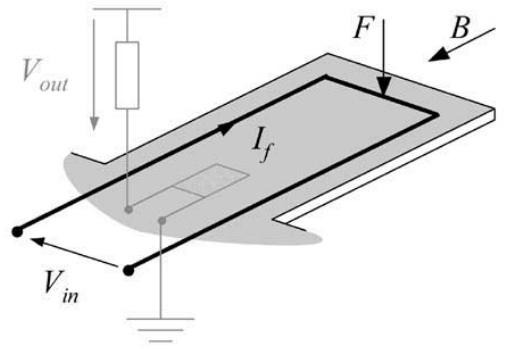

(b)

Fig. 1. (a) Generic cantilever-based transducer. (b) Application to electromechanical filtering.

order to write an HDL model for a transducer, one should first describe the behavior of the structure with a set of equations (similar to one of an MOS transistor). Such a set of equations may result from finite-element parametric analysis and interpolation [3], [4]. This is often the only solution to handle complex systems. For simpler structures, the modeling can be conducted analytically by using well-established results from the concerned scientific fields. In this case (i.e., simpler structures), physical parameters such as technological constants or device dimensions are directly part of equations, leading to a faster, intuitive optimization method [5].

In this paper, we address the design flow of a monolithic MEMS through an alternative approach to dedicated MEMS oriented tools. Our main focus is the electromechanical part modeling and description, the system description and the system level simulation and verification. The studied CMOS MEMS is composed of a mechanical resonator acting as a highly selective pass-band filter, a frequency shifter, plus some standard analog elements for signal amplification and filtering. Mechanical filters feature large quality factors in comparison with standard electronic solutions [6]-[8].

The first section discusses the principle and modeling of the mechanical filter. Although most of the modeling results are not new, equations are recalled in order to provide a full understanding of the HDL code provided in the second section. After a presentation of the complete system in the third section, the fourth and final section is devoted to simulation results using a recently released CAD tool to handle multifrequency simulation problems.

\section{Mechanical Filter: Principle AND Modeling}

The mechanical filter is based on a generic cantilever device which is presented in Fig. 1(a). It is made of a simple beam that converts the applied force $F$ into a bending $z$. The latter produces a resistance change $\Delta R$ in an embedded piezoresistive polysilicon strain gauge. The gauge resistance variation can then be converted into an electrical signal $V_{\text {out }}$ by means of a simple resistor bridge (or a Wheatstone bridge).

In this example, the beam is actuated by the Lorentz force [Fig. 1(b)], resulting from the interaction between an electrical current $I_{f}$ flowing into the structure and a magnetic field. The external dc magnetic field 


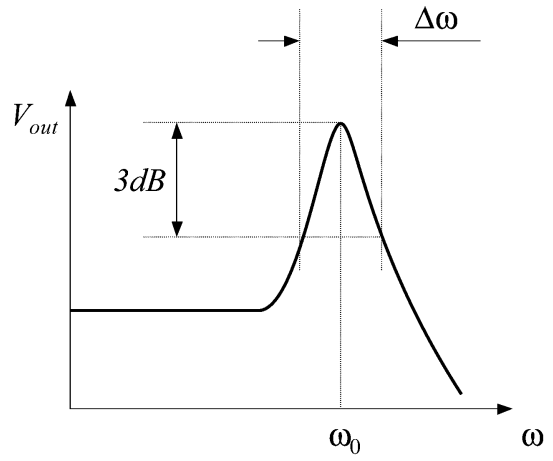

Fig. 2. Typical frequency response of a low-damped, second-order filter.

is generated by a permanent magnet, simply glued onto the device package. For signal filtering applications, the input signal $V_{\text {in }}$ is the voltage applied across the integrated planar metal coil in which the current $I_{f}$ flows.

Given the resistance $R_{f}$ of this coil, the electrical current $I_{f}$ can be easily calculated. The applied force is written

$$
F=\frac{V_{\text {in }}}{R_{f}} \cdot B \cdot W c .
$$

\section{A. Static Behavior}

First-order modeling of the static behavior of the cantilever beam is the well-known spring law that links the applied force $F$ to the induced bending $z$

$$
F=k \cdot z
$$

where $k\left(\mathrm{~N} \cdot \mathrm{m}^{-1}\right)$ is the cantilever stiffness. The bending $z$ causes the gauge to stretch. Due to the piezoresistive properties of polysilicon, the gauge resistance varies linearly with the bending as follows:

$$
\frac{\Delta R}{R}=T_{\mathrm{GF}} \cdot k \frac{L_{C}}{W_{C}} \cdot z
$$

where $T_{\mathrm{GF}}$ is an electromechanical transfer coefficient that depends on process parameters such as vertical dimensions and the polysilicon gauge factor.

\section{B. Dynamic Behavior}

For dynamic behavior, the cantilever structure is modeled by a wellknown second-order mechanical system. It features a mass $M$, the previous spring $k$ and a damping factor $D$. The response of the mechanical system to the force $F$ is therefore described by the differential equation

$$
M \frac{d^{2} z}{d t^{2}}=-k z-D \frac{d z}{d t}-F .
$$

The frequency response of such a system is illustrated in Fig. 2. Because of the large resonance phenomenon, it is possible to use such a system as a band-pass filter. For low-damped systems, the filter's central frequency is then very close to the mechanical natural frequency, which is defined by

$$
f_{0}=\frac{\omega_{0}}{2 \pi}=\frac{1}{2 \pi} \sqrt{\frac{k}{M}} .
$$

Another important feature of the resulting band-pass filter is the -3-dB bandwidth. This bandwidth $\Delta \omega$ depends on both the resonant frequency and the quality factor as given by

$$
Q=\frac{\omega_{0}}{\Delta \omega}
$$

\section{Noise Modeling}

The major source of noise in resistive strain gauges is thermal noise, which appears as a white noise. The noise spectral density is therefore a constant over the frequency range, given by

$$
V_{\text {noise }}^{2}(f)=4 k_{B} T R \quad\left[\frac{V^{2}}{H z}\right]
$$

where $k_{B}=1.38 \times 10^{-23} J K^{-1}$ is the Boltzmann's constant, $T$ is the temperature in Kelvin and $R$ is the resistance in ohms.

In addition, small moving parts are susceptible to mechanical noise resulting from molecular agitation of the surrounding gas. This thermal-mechanical noise, which is physically equivalent to Johnson noise in resistors, can be modeled by adding a random force generator alongside each damper [9]. From the fluctuation-dissipation theorem [10], the spectral density of the fluctuating force is given by

$$
F_{\text {noise }}^{2}(f)=4 k_{B} T D \quad\left[\frac{N^{2}}{H z}\right] .
$$

\section{MechaniCAL Filter: Model VALIDATION AND INTEGRATION}

The use of a standard microelectronic technology for MEMS applications presents several advantages in terms of cost or monolithic integration. However, process parameters delivered by the CMOS foundry, mainly concern electronic design. Fortunately these also include back-end layer thickness which is useful for mechanical applications. In contrast, material mass-densities or Young's modulus are not characterized. A characterization methodology has therefore been developed in order to measure the necessary parameters (basically $k$, $M, D$, and gauge factor) and to validate the model of the mechanical filter. After validation, the model is described in an analog HDL language to be implemented in a standard microelectronic CAD tool.

\section{A. Model Validation}

The characterization methodology is based on the experimental study of a set of specific test vehicles called "U-Shape" cantilevers, presented in Fig. 3. This cantilever device can be actuated by a test-probe or by using the Lorentz force, which allows both static and dynamic characterization. The whole characterization methodology has been reported in detail [11], along with the experimental results, which were obtained on a set of 25 chips.

Based on test results, the unknown technological parameters have been extracted, thus allowing for comparison between experimental and modeled cantilever behavior. An example of such a comparison is given in Fig. 4, where the experimental step response is used to validate the second order model assumption using the overshoots envelope. This $O_{n}$ envelope is calculated using the well-known relation

$$
\ln \frac{O_{1}}{O_{n}} \approx(n-1) \frac{D}{2 M f_{0}} .
$$

The close match between experiment and theory validates the second order system assumption for the model. Furthermore, it demonstrates the correct values of the main filter parameters (stiffness, mass, and damping factor).

Finally, note that the proposed model is valid for small displacements of the cantilever end. A nonlinear mechanical behavior is observed when the cantilever is actuated in such a way that oscillation amplitude reaches about $10 \%$ of the beam length. Such conditions have been obtained at resonance and under vacuum conditions, where damping forces are dramatically reduced. 


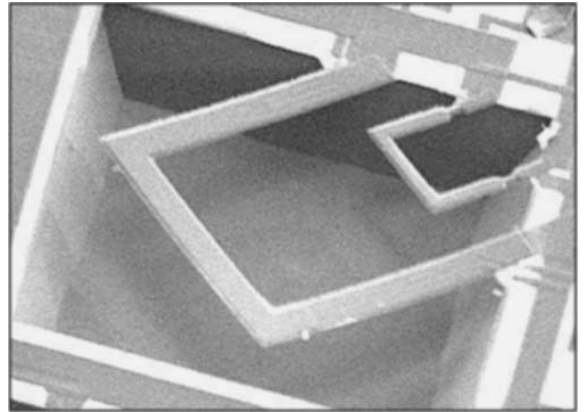

Fig. 3. SEM picture of the fabricated test-vehicle (U-Shape cantilever).

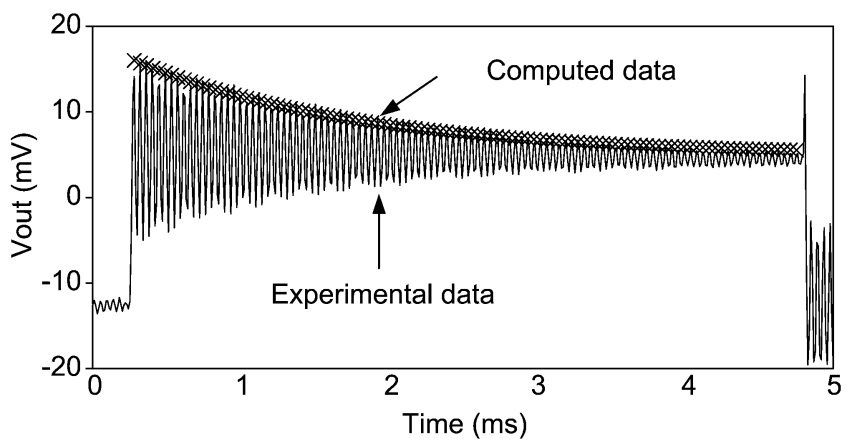

Fig. 4. Comparison of calculated and experimental cantilever step response for model validation.

\section{B. Model Integration in a Microelectronic CAD Tool}

The model of the mechanical filter is translated into a Verilog-A module, whose listing is given in Fig. 5. The ports are, for inputs: the coil terminals $(f a, f b)$ and the external magnetic field $(B)$; and for outputs: the strain gauge terminal $(r a, r b)$. The bending $z$ is added at the module interface for debug purposes only. A default cantilever design (i.e., for one fixed dimension) is defined by initializing the model parameters. However, it is possible to change these values before simulation by editing the filter properties directly in the schematic view and then adapting the model for various designs or operating conditions.

The filter behavior is described in the "analog" section. It includes: 1) the calculation of the Lorentz force, with a given input voltage; 2) the differential equation set for the mechanical system; and 3) the Ohm's Law applied across the piezoresistive gauge. Electrical and mechanical noise sources are also described according to (7) and (8).

\section{SySTEM ARCHITECTURE}

In order to validate the design flow for monolithic MEMS, we have defined a minimal system including both electronics and mechanical parts. The studied system is based on the swept frequency, super-heterodyne spectrum analyzer [12], whose simplified block diagram is given in Fig. 6 . The signal $V_{\text {meas }}$ to be analyzed is first multiplied by a frequency swept signal $V_{\mathrm{fs}}$. The input signal is thus shifted in the frequency domain. Each time the frequency of the mixer output $V_{s}$ matches the central frequency $\omega_{0}$ of the band pass filter, an ac voltage peak appears at the filter output. Since both the filter characteristics and the frequency of $V_{\mathrm{fs}}$ are known, the input signal spectrum can be easily reconstructed.

This spectrum analyzer features an electronic mixer and benefits from the high-Q mechanical filtering introduced in the previous sections.

\section{A. Mixer}

A Gilbert cell has been used to implement the mixer. As per Fig. 7, this mixer is made of a differential amplifier stage based on $\mathrm{T} 1$ and $\mathrm{T} 2$, with $V_{\text {meas }}$ as input. Depending on the control signal $V_{\mathrm{fs}}$ applied on transistors T3 to T6, the amplifier gain can be turned from positive $A$ to negative $-A$. Although not a pure multiplier, such an architecture provides the required frequency shift. The mixer output spectrum also exhibits harmonic tones that result from the harmonics of the square control signal $V_{\mathrm{fs}}$.

\section{B. Filter Performance}

Quality factors greater than 100 have been measured on fabricated cantilevers featuring a $8.9-\mathrm{kHz}$ resonant frequency [11]. This leads to a filter bandwidth in the $100 \mathrm{~Hz}$ range, which is a good performance in comparison with integrated electronic filters. Fig. 8 compares the quality factor of such a cantilever operating in air and under vacuum conditions. In vacuum, the beam-damping factor is drastically reduced, and a quality factor of about 4000 has been observed. The corresponding $-3 \mathrm{~dB}$ bandwidth is then approximately $3 \mathrm{~Hz}$, making such a filter suitable for highly selective filtering applications.

\section{SySTEM-LEVEL SimUlations}

As previously mentioned, analog HDL languages allow us to describe multidomain systems with a microelectronic CAD tool. As long as the device is defined using a consistent unit system, the electrical simulator handles real numbers regardless of their physical significance. For this reason, a voltage generator can be used to emulate various stimuli such as magnetic field input. Note that the Verilog-A language authorizes the definition of various disciplines (kinematic, magnetic...) for clarity of the written code.

A second specificity of our system relates to the multifrequency domain. The mixer inputs receive signals of different frequencies. In addition, the settling time of the mechanical filter, when operated under vacuum conditions, is in the range of several hundreds of milliseconds, while the resonator oscillates at about $10 \mathrm{kHz}$. The simulation time step should then be kept small enough to accurately simulate transient response (with respect to the oscillation frequency) over a long time frame (with respect to the settling time). Calculating transient steady-state for such a resonator thus requires unacceptable simulation time. Finally, traditional simulators perform small-signal analysis around a dc operating point, which is not adequate for the study of transfer functions that include frequency shifts.

In order to validate the proposed spectral analyzer and to demonstrate the efficiency of mechanical filtering, we have chosen to analyze the spectrum of a $50 \mathrm{~Hz}$ square-wave signal applied to the $V_{\text {meas }}$ input. Among the required system level analyzes and verifications, three are not possible (or are time consuming) in a classical electrical simulator:

- a periodic steady state analysis, to measure the selectivity of the system;

- a periodic small-signal analysis, to retrieve the spectrum of the input signal;

- a periodic noise analysis, to evaluate the system resolution

Therefore, we used the recently released Spectre-RF simulator that supports small-signal analysis of multifrequency systems and more generally, of any periodically time-varying system, such as mixers or switched-capacitor filters, regardless of their operating frequencies [13]. The simulator comes with an analysis suite, that includes: periodic steady state (PSS), which can speed up the steady-state calculation for systems having large settling times; periodic ac (PAC), periodic transfer function (PXF), and periodic noise analysis (PNOISE), which performs small-signal analysis based on a periodically time-varying operating point. 


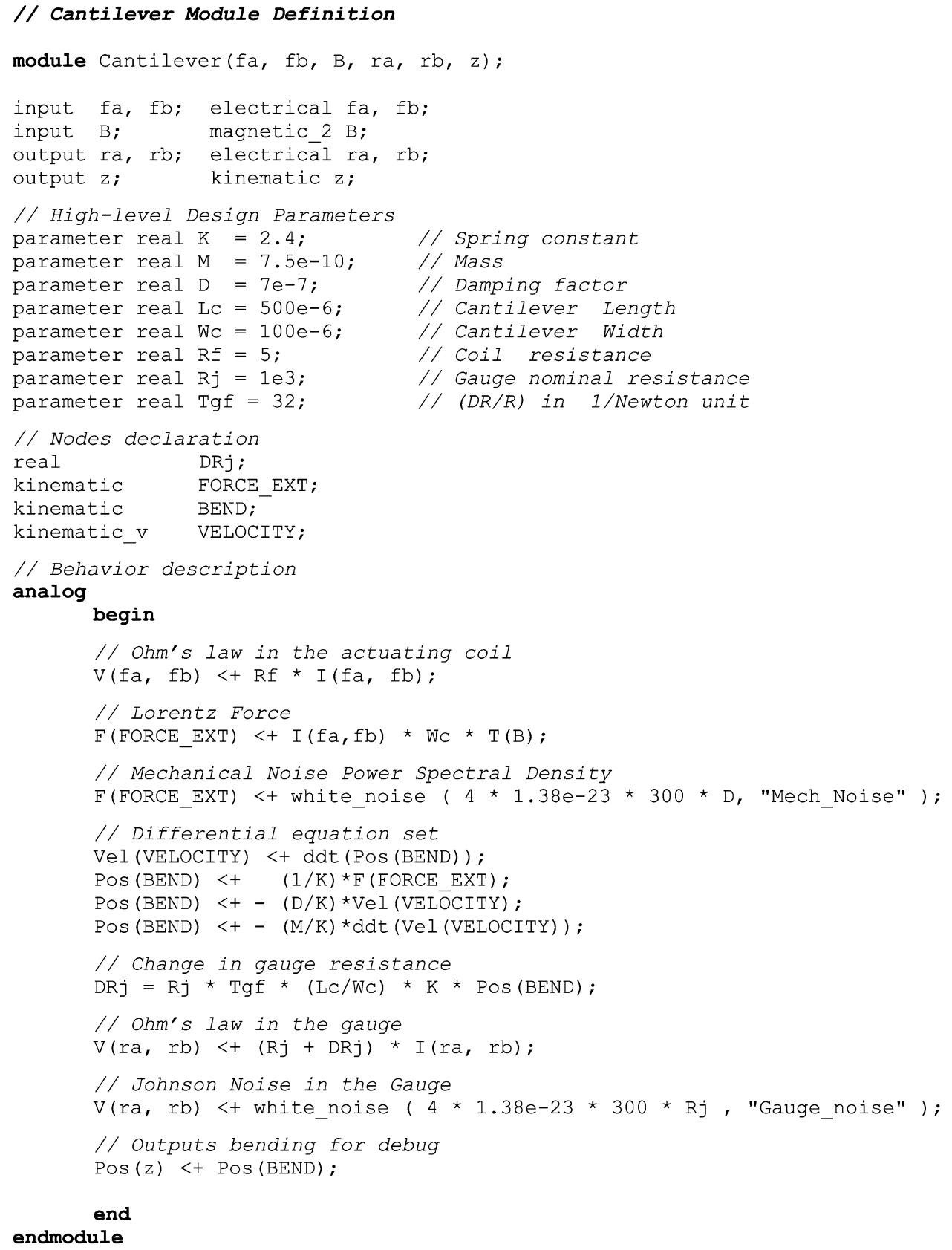

Fig. 5. Verilog-A description of the cantilever used as a mechanical filter.

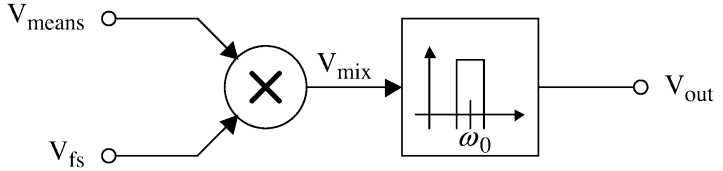

Fig. 6. Simplified spectrum analyzer block diagram.

Regarding the test bench considered in the following, the mechanical filter features a $9-\mathrm{kHz}$ resonant frequency and $-3-\mathrm{dB}$ bandwidth of $3 \mathrm{~Hz}$. This corresponds to a realistic device of $500 \times 160 \mu \mathrm{m}$, packaged under vacuum.

\section{A. PSS Analysis}

The PSS analysis computes the system steady-state in both time and frequency domains. In our case, a single $9.05-\mathrm{kHz}$ signal is applied on $V_{\mathrm{fs}}$. Once mixed with the analyzed $50-\mathrm{Hz}$ square-wave signal, the main frequency components at the filter input are then located at 9 and $9.1-\mathrm{kHz}$. The PSS analysis enables us to study the output spectrum of the system (Fig. 9) and to demonstrate the rejection of the $9.1-\mathrm{kHz}$ component, thus verifying the selectivity of the filter. As a result of the selective cantilever filtering, the $9.1-\mathrm{kHz}$ tone is $30 \mathrm{~dB}$ below the $9-\mathrm{kHz}$ tone. This attenuation is consistent with the characterized frequency response of the mechanical filter presented in Fig. 8 (vacuum condition).

This analysis could also be performed using standard electrical-simulation analysis, which consists of a transient analysis, plus a FFT of the output signal. However, the multifrequency domain implies that the steady-state be reached before performing the analysis. For this reason, the transient analysis should be performed over a long time period to reach the steady-state. Simulation times may then increase drastically.

Regarding simulator convergence, it is worth noting that the integration method should be chosen carefully for low-damped system transient simulation. In this case, an integration algorithm based on 


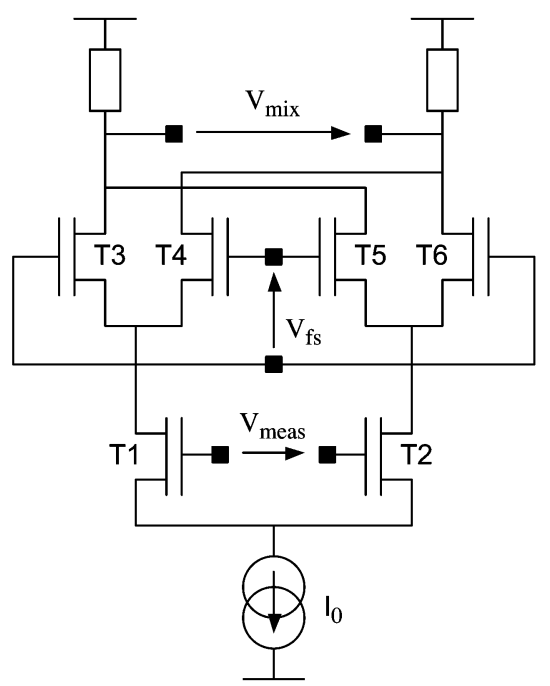

Fig. 7. The Gilbert cell based switching mixer.

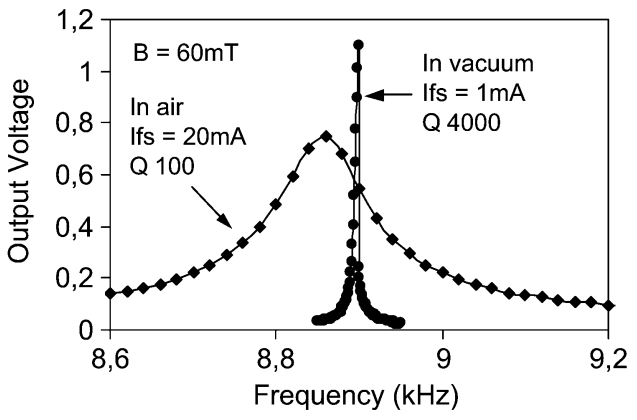

Fig. 8. Measurement of cantilever quality factors for operation in air and under vacuum conditions.

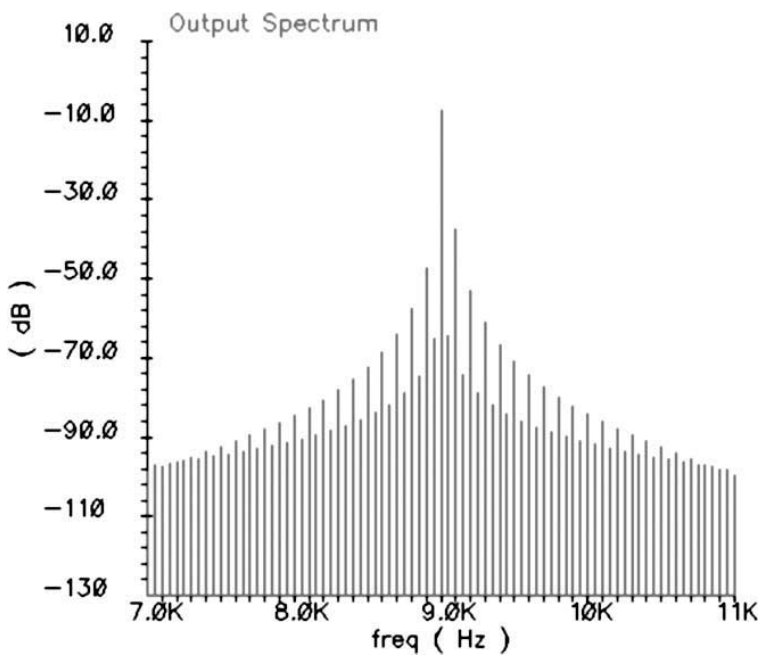

Fig. 9. PSS analysis result. Spectrum of the output signal.

trapezoidal approximations gives good results with respect to the experiment. This is not the case when using the Euler integration method.

\section{B. PAC Response (PAC Analysis)}

The PAC analysis consists of a multifrequency analysis. In our case, we performed a small-signal analysis at the mixer input $V_{\mathrm{fs}}$ while the square signal $V_{\text {meas }}$ was applied at a constant $50-\mathrm{Hz}$ frequency. For this analysis, no dc point, but a whole transient

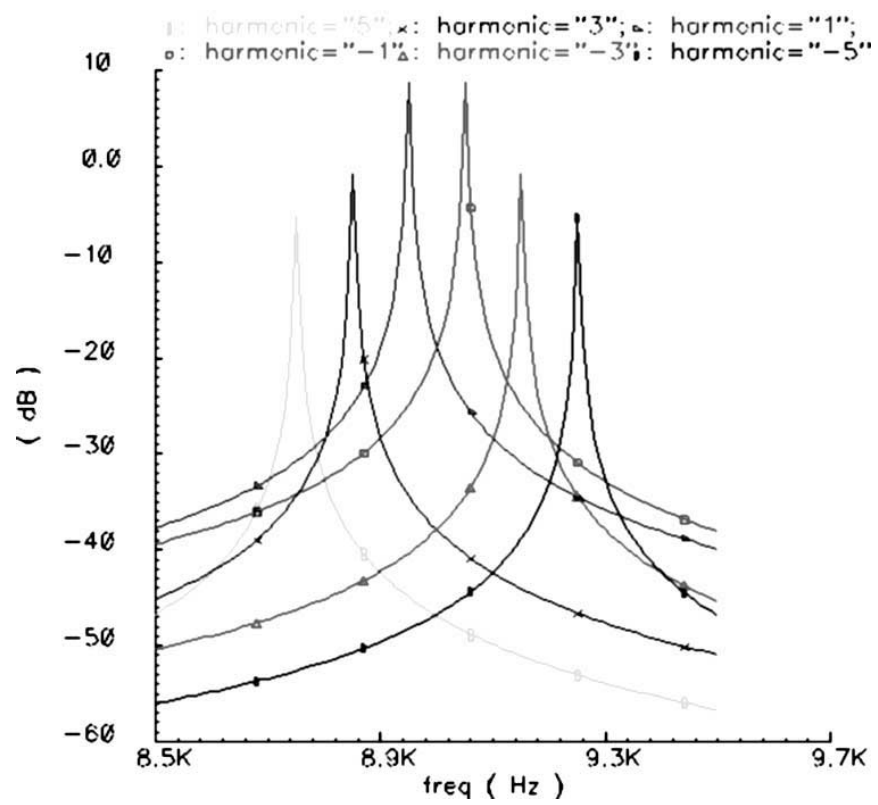

Fig. 10. PAC analysis result ( $X$ axis $=$ output frequency $)$.

period of $20 \mathrm{~ms}$ was considered. Transfer functions (similar to standard ac analyses) were then computed for each harmonic of the 50-Hz signal (i.e., for each $n \times F_{\text {fund }}$, with $F_{\text {fund }}=50 \mathrm{~Hz}$, and $n=\left[-n_{\max }, \ldots,-2,-1,1,2, \ldots, n_{\max }\right]$. Note that this analysis has no experimental equivalent since the $50 \mathrm{~Hz}$ harmonics are considered separately.

The results of a PAC analysis are shown in Fig. 10. The $x$ axis represents the specified input frequency range $\left(V_{\mathrm{fs}}\right)$, which has been set from $8.5 \mathrm{kHz}$ to $9.5 \mathrm{kHz}$. A number of 5 input sidebands $\left(n_{\max }=5\right)$ were analyzed (in order to limit the simulation time to few minutes). The obtained curves are interpreted as follows: when mixed with the $V_{\text {meas }}$ fundamental tones $( \pm 50 \mathrm{~Hz})$, the maximum output signal is found at input frequencies of $8.95 \mathrm{kHz}$ (harmonic " 1 ") and $9.05 \mathrm{kHz}$ (harmonic "-1"); when mixed with $V_{\text {meas }}$ third order harmonics $( \pm 150 \mathrm{~Hz})$, the maximum output signal is found at input frequencies of $V_{\mathrm{fs}}$ at $8,85 \mathrm{kHz}$ (harmonic " 3 ") and $9.15 \mathrm{kHz}$ (harmonic “-3"); and so on. Transfer functions for even order harmonics (i.e., $\pm 100 \mathrm{~Hz}$ for $n= \pm 2, \pm 200 \mathrm{~Hz}$ for $n= \pm 4 \ldots$ ) were computed as well and give results far below the $-60-\mathrm{dB}$ lower limit of the $y$ axis.

This simulation qualitatively verifies that the proposed system performs the required spectrum analysis. Indeed, the $V_{\text {meas }}$ signal spectrum can be easily extrapolated from the PAC results, by summing up all the harmonic contributions and shifting down the spectrum by $9 \mathrm{kHz}$ (the band-pass filter cutoff frequency).

If the output frequency is selected for the PAC $x$ axis instead of the input frequency, the result represented in Fig. 11 is obtained. Only 9-kHz tones are observed, as expected after mechanical filtering. It again appears that the system response is maximal for the fundamental $50-\mathrm{Hz}$ tone. Next are the $50-\mathrm{Hz}$ third-order harmonic $(150 \mathrm{~Hz})$ and the fifth-order harmonic $(250 \mathrm{~Hz})$. Let us use this simulation result to quantitatively evaluate the spectrum retrieval.

Assuming a square-wave $S$ of amplitude 1, attenuations of the successive harmonics are given by the Fourier transform

$$
S(t)=\frac{4}{\pi} \sum_{n=0}^{n=\infty} \frac{(-1)^{n}}{2 n+1} \cos \left[2 . \pi(2 n+1) f_{\text {fund }} . t\right]
$$

where $n$ denotes the harmonic index. For $n=1(150 \mathrm{~Hz})$, an attenuation of $1 / 3$ corresponds to approximately $-10 \mathrm{~dB}$ and for $n=2$ 


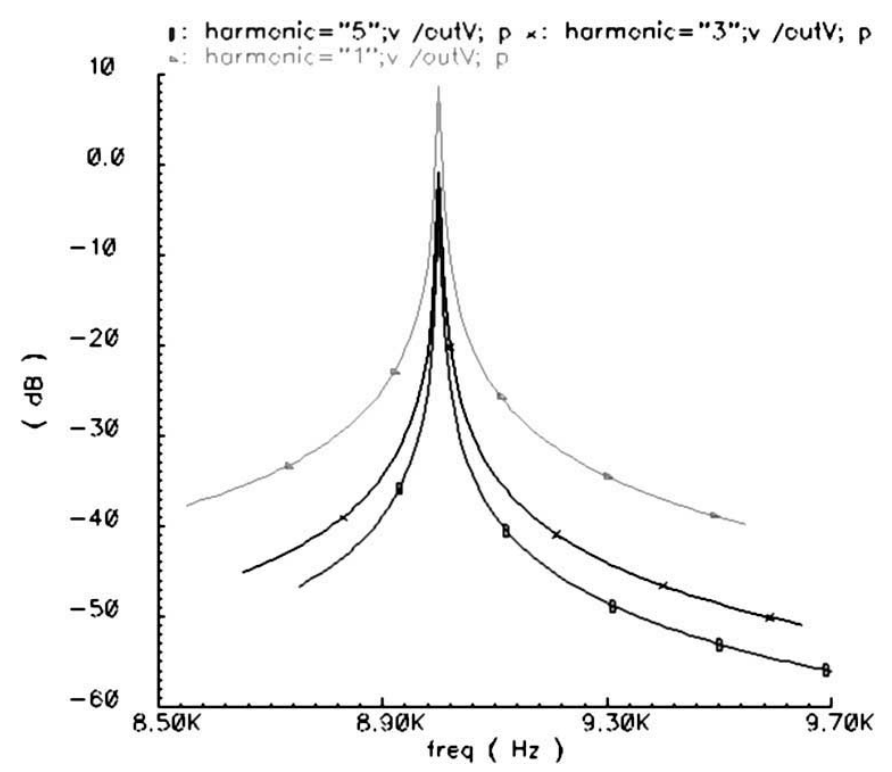

Fig. 11. PAC analysis result ( $X$ axis $=$ output frequency $)$.

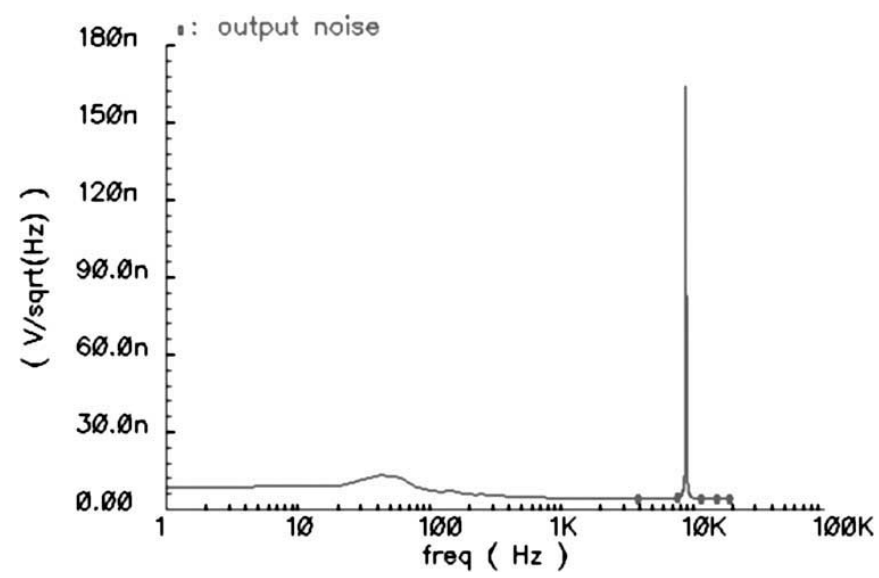

Fig. 12. PNOISE analysis result: noise spectral density in the system-output signal.

$(250 \mathrm{~Hz})$, an attenuation of $1 / 5$ corresponds to approximately $-14 \mathrm{~dB}$. According to Fig. 11, simulation results comply with theory.

\section{PNOISE}

The PNOISE analysis is a small-signal analysis that computes noise effects, taking into account both folding, aliasing, and frequency-conversion phenomenon. In our case, the $1 / f$ noise generated by the mixer is translated in the frequency domain by the $V_{\text {meas }}$ signal and its harmonics, before being shaped by the filter-transfer function. This is precisely the result verified by PNOISE analysis, which computes the noise spectral density of the system-output signal (Fig. 12). This analysis may therefore be used by system designers to evaluate the resolution of the system with respect to the signal to noise ratio.

\section{CONCLUSION}

In this paper, we have presented the use of a standard microelectronic CAD tool to implement the design flow of a MEMS. The system used to illustrate the methodology is a spectrum analyzer based composed of a mechanical filter and an electronic mixer for frequency sweep purposes. The first step of the design approach consists of characterizing the mechanical device to produce a parameterized model that can be described in an analog HDL language such as Verilog-A. The mechanical device can then be handled as a classical parameterized cell independently of the physical nature of the cell inputs. This allows multidomain simulations using a SPICE-like simulator. The second contribution of this paper is the illustration of the recently released Spectre-RF simulator that supports small-signal analysis for multifrequency systems such as mixers regardless of their operating frequencies. Several analyses were performed on the spectrum analyzer to validate the spectral analysis of a low-frequency $(50 \mathrm{~Hz})$ square-wave signal. Based on small-signal periodic ac analysis results, the square-wave signal harmonics separated by only $100 \mathrm{~Hz}$ were identified with a very good resolution, as a result of the selectivity of the mechanical filter.

Finally, the use of a standard microelectronic CAD tool for MEMS design has been validated. Such a design methodology is currently used in our laboratory for the design of MEMS, including a magnetic field sensor for navigation systems [14].

\section{ACKNOWLEDGMENT}

The authors would like to acknowledge A. B. Desormeaux from Cadence Design Systems SAS, for his support in modeling and simulations.

\section{REFERENCES}

[1] G. Zhou and P. Dowd, "Modeling and simulation of micro-electromechanical systems with an Analog Hardware Description Language," in Proc. Transducers'01 Eurosensors XV, Munich, Germany, June 10-14, 2001, pp. 268-271.

[2] E. Christen and K. Bakalar, "VHDL-AMS-A hardware description language for analog and mixed-signal applications," IEEE Trans. Circuits Syst. II, vol. 46, pp. 1263-1272, 1999.

[3] M. Varghese, S. D. Senturia, J. R. Gilbert, and V. Rabinovich, "Automatic reduced-order modeling in MEMCAD using modal basis functions," in Proc. Transducers'01 Eurosensors XV, Munich, Germany, June 10-14, 2001, pp. 264-267.

[4] J. V. Clark, D. Bindel, W. Kao, E. Zhu, A. Kuo, N. Zhou, J. Nie, J. Demmel, Z. Bai, S. Govindjee, K. S. J. Pister, M. Gu, and A. Agogino, "Addressing the needs of complex MEMS design," in Proc. IEEE MEMS'02, Las Vegas, NV, Jan. 20-24, 2002, pp. 204-209.

[5] L. Latorre, P. Nouet, Y. Bertrand, P. Hazard, and F. Pressecq, "Characterization and modeling of a CMOS-compatible MEMS technology," Sens. Actuators A, vol. 74/1-3, pp. 143-147, 1999.

[6] R. A. Johnson, Mechanical Filters in Electronics. New York: Wiley, 1983.

[7] D. Lange, C. Hagleitner, C. Herzog, O. Brand, and H. Baltes, "Magnetic actuation and MOS-transistor sensing for CMOS-integrated resonators," in Proc. IEEE MEMS'02, Las Vegas, USA, Jan. 20-24, 2002, pp. 304-307.

[8] L. Liwei, R. T. Howe, and A. P. Pisano, "Microelectromechanical filters for signal processing," J. Microelectromech. Syst., vol. 7, pp. 286-294, Sept. 1998.

[9] T. B. Gabrielson, "Mechanical-thermal noise in micromachined acoustic and vibration sensors," IEEE Trans. Electron Devices, vol. 40, pp. 903-909, May 1993.

[10] H. B. Callen and T. A. Welton, "Irreversibility and generalized noise," Phys. Rev., vol. 83, no. 1, pp. 34-40, 1951

[11] L. Latorre, V. Beroulle, M. Dardalhon, P. Nouet, F. Pressecq, and C. Oudea, "Characterization of CMOS MEMS technology scatterings," in Proc. Int. Symp. Test Failure Anal., Santa Clara, CA, Nov. 11-15, 2001, pp. 373-377.

[12] IEEE Std. Spectrum Analyzers, IEEE Std 748-1979.

[13] Cadence SpectreRF Tools, Lecture Manual, Version 4.4.6, 2001.

[14] V. Beroulle, Y. Bertrand, L. Latorre, and P. Nouet, "Micromachined CMOS magnetic field sensors with low-noise signal conditioning," in Proc. IEEE MEMS'02, Las Vegas, NV, Jan. 20-24, 2002, pp. 256-259. 